\title{
Multichannel Segmentation Using Contour Relaxation: Fast Super-Pixels and Temporal Propagation
}

\author{
Rudolf Mester $^{1,2}$, Christian Conrad ${ }^{1}$, and Alvaro Guevara ${ }^{1}$ \\ 1 VSI Lab*, Computer Science Dept., Goethe University, Frankfurt, Germany \\ ${ }^{2}$ Computer Vision Laboratory, Dept. EE, Linköping University, Sweden
}

\begin{abstract}
The contribution describes a statistical framework for image segmentation that is characterized by the following features: It allows to model scalar as well as multi-channel images (color, texture feature sets, depth, ...) in a region-based manner, including a Gibbs-Markov random field model that describes the spatial (and temporal) cohesion tendencies of 'real' label fields. It employs a principled target function resulting from a statistical image model and maximum-a-posteriori estimation, and combines it with a computationally very efficient way ('contour relaxation') for determining a (local) optimum of the target function. We show in many examples that even these local optima provide very reasonable and useful partitions of the image area into regions. A very attractive feature of the proposed method is that a reasonable partition is reached within some few iterations even when starting from a 'blind' initial partition (e.g. for 'superpixels'), or when — in sequence segmentation the segmentation result of the previous image is used as starting point for segmenting the current image.
\end{abstract}

\section{Motivation}

The term 'image segmentation' is used in a very large variety of meanings; in the ideal case it denotes the process of subdividing images into 'meaningful' regions; however, what the term 'meaningful' means is hard to pinpoint. For recent reviews, see e.g. [16]5. If we use the term 'segmentation' in the following, we intend to refer to the computation of a division of a given image into parts that are homogeneous from a signal perspective, that is: homogeneous w.r.t. a statistical model of color, gray value, depth or similar 'features' that can be associated with a pixel, or pixel cell, on the image grid. We present an approach that is able to compute such an 'over-segmentation', particularly for the case of multichannel data (color, or gray value plus other features). As Veksler et al [17] state, the idea of operating on atomic, homogeneously colored or textured regions is old (see for instance [11] as only one example), whereas the now popular

\footnotetext{
* This work was funded by the German Federal Ministry of Education and Research (BMBF) in the project Bernstein Fokus Neurotechnologie -01GQ0841, and in parts supported by the ELLIIT programme funded by the Swedish Government.
} 
term 'superpixels' has been coined recently [12] and is a very active research area [79117]. We consider it to be the main characteristics of our approach that it is statistically principled and offers a really quick transition from a 'bad' partition (that could be 'guessed' or determined by some heuristic) into a very good oversegmentation.

\section{The Region-Based Generative Image Model for Multi-channel Data}

A region-based image model assumes that the structure of each image is dominated by regions. The complete image area is subdivided into a partition $\mathcal{Q}$ consisting of regions $R_{i}, i=1, \ldots, N_{R}$. Each site on the image grid is associated with a measurable entity, for instance a gray value, or an RGB color value. It might also be that additional entities which are not directly measurable are associated with the individual grid sites, for instance a motion vector, a disparity vector, a depth value, or combinations of such entities. Finally, a region could also be filled by values which are determined from intermediate computations, e.g. the output of a set of texture operators. In the interest of a compact presentation, we denote all these variants as the (region-specific) texture signal.

Besides that each measurement may be single channel or vectorial (= multichannel), measurements can be organized in cells (see Fig.21), for instance for implementing multi-resolution segmentation algorithms. We do not discuss cells here due to limited space; the mathematical generalization is straightforward.

It is important that a probability measure can be associated with each realization of the texture signal, that is: it is considered as a realization of a random process. These region-specific random processes share the same functional structure all over the image, but each region is associated with an individual region-specific parameter vector $\boldsymbol{\theta}_{i}$ (see Fig.11). So only the numerical values of these parameters vary from region to region.

In the experiments performed in this paper, we make two assumptions which drastically simplify the appearing expressions and reduce the computational effort, being fully aware that both these assumptions are not true for real image material. However, we show that even these drastic approximations do not prevent the resulting procedures from yielding very useful results. It has been confirmed in a very large number of experiments that even a very shallow, incomplete description of region texture signals is most of the time sufficient for differentiating such textures. These approximations are:

A1: The feature values at different pixel sites obey the same distribution inside of a region, but they are statistically independent of each other.

A2: The feature values in the different feature channels at each pixel site are statistically independent of each other. 


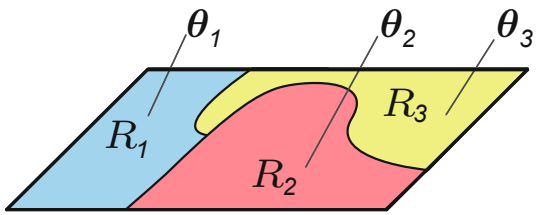

Fig. 1. Region-based image model, associating one parameter vector $\boldsymbol{\theta}_{i}$ to each region $R_{i}$. The values at each pixel site can be vector-valued (=multi-channel).

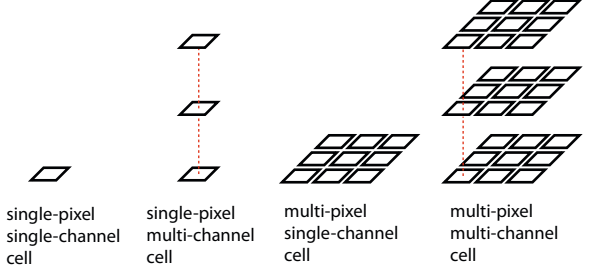

Fig. 2. Measurements are organized in cells which may include a single measurement or several ones; each measurement may be single channel or vectorial (= multi-channel)

\section{Segmentation as a Maximum-a-Posteriori (MAP) Estimation Problem}

We proceed by formulating the segmentation task as an estimation problem, as this deduces an 'energy function' to be optimized from first principles. Corresponding approaches can already be found in early publications on image segmentation such as [15/14/46/11.

Let $\boldsymbol{z}$ be the vector of measurements (a $2 \mathrm{D}$ array of vectors) on the image array and let $\mathcal{Q}=\left\{R_{1}, R_{2}, \ldots R_{n}\right\}$ be a partition of the image array. We assume that the measurements inside of each region have been generated by a regionspecific stochastic process. Each of these processes is characterized by a set of parameters. Thus, to each region $R_{i}$ of the partition, a stationary stochastic process with an individual model parameter vector $\boldsymbol{\theta}_{i}=\boldsymbol{\theta}\left(R_{i}\right)$ is assigned.

The ensemble $\{\boldsymbol{\theta}\}=\left\{\boldsymbol{\theta}_{1}, \boldsymbol{\theta}_{2}, \ldots \boldsymbol{\theta}_{n}\right\}$ of model parameter vectors $\boldsymbol{\theta}_{i}$ represents, together with the partition $\mathcal{Q}$, a complete, piecewise stationary 11 model for the image process. We denote the combination of a partition $\mathcal{Q}$ and the parameter ensemble $\{\boldsymbol{\theta}\}$ as the 'array state' $\mathcal{S}$. For each completely specified array state $\mathcal{S}$, we can provide a conditional probability density for the total ensemble of random variables $\boldsymbol{z}$, conditioned on $\mathcal{Q}$ and $\{\boldsymbol{\theta}\}$. With prior distributions for the partition $\mathcal{Q}$ and the parameter set $\{\boldsymbol{\theta}\}$, we can turn this into a joint distribution $p(\boldsymbol{z}, \mathcal{Q},\{\boldsymbol{\theta}\})=p(\boldsymbol{z}, \mathcal{S})$.

Based on this formulation, the segmentation task now consists in finding a likely (hidden) array state $\mathcal{S}$ as the cause for an observed image vector $\boldsymbol{z}$.

In maximum-a-posteriori (MAP) segmentation, we consider the specific combination of a partition $\mathcal{Q}$ and the corresponding model parameters $\left\{\boldsymbol{\theta}_{1}, \boldsymbol{\theta}_{2}, \ldots \boldsymbol{\theta}_{n}\right\}$ as the sought estimate of the array state $\mathcal{S}$ if it maximizes the posterior distribution

$$
p(\mathcal{S} \mid \boldsymbol{z})=p\left(\mathcal{Q},\left\{\boldsymbol{\theta}_{1}, \boldsymbol{\theta}_{2}, \ldots \boldsymbol{\theta}_{n}\right\} \mid \boldsymbol{z}\right) .
$$

\footnotetext{
${ }^{1}$ We can deviate from stationarity by assuming a smooth deterministic parametric function to be overlaid by a stationary process, but this is not done here.
} 
This posterior is, as always, linked by Bayes' theorem with the prior and the conditional observation density:

$$
p(\mathcal{S} \mid \boldsymbol{z})=p(\boldsymbol{z} \mid \mathcal{S}) \cdot p(\mathcal{S}) / p(\boldsymbol{z})
$$

Since the observed image vector $\boldsymbol{z}$ is a fixed realization of a random process, $p(\boldsymbol{z})$ is merely a normalization constant. Thus the MAP segmentation is given by the particular array state $\mathcal{S}$ which maximizes

$$
p(\boldsymbol{z} \mid \mathcal{S}) \cdot p(\mathcal{S})=p(\boldsymbol{z}, \mathcal{S})=p(\boldsymbol{z} \mid \mathcal{Q}, \boldsymbol{\theta}) \cdot p(\boldsymbol{\theta} \mid \mathcal{Q}) \cdot p(\mathcal{Q}) \quad \rightarrow \max \quad .
$$

This way we try to find the 'most probable cause' for the generation of the given image. Now we have to specify the conditional densities for $\boldsymbol{z}$, given the individual region model processes, and also the prior distribution $p(\mathcal{Q})$ for the partitions $\mathcal{Q}$. Gibbs random fields $(\mathrm{GRF})$ relate $p(\mathcal{Q})$ to the clique potentials $V_{c}\left(c_{i}\right)$ defined on pairs of neighboring sites on the label array. These 'potentials' depend on whether the label values in such a clique are identical or not. The sought distribution is built from the clique potentials as

$$
p(\mathcal{Q})=\frac{1}{Z} \cdot \exp \left(-\sum_{c_{i}} V_{c}\left(c_{i}\right)\right) .
$$

Fortunately, the 'partition function' $Z$ in Eq. 4 is not needed in our algorithm.

The remaining entity to be specified are the region-specific model parameter vectors $\boldsymbol{\theta}$. They are considered as unknown 'deterministic parameters', that is: there is no non-uniform prior on their value range. With that assumption, we can write

$$
p(\mathcal{S})=p(\boldsymbol{\theta}, \mathcal{Q})=k \cdot p(\mathcal{Q}) .
$$

The model parameters $\boldsymbol{\theta}$ are obtained by a region-specific maximum likelihood estimation: the partition $\mathcal{Q}$ is kept fixed, and the term $p(\boldsymbol{z} \mid \mathcal{S})$ is maximized with respect to the parameter $\boldsymbol{\theta}$

$$
\begin{aligned}
p(\boldsymbol{z}, \mathcal{S}) & =p(\boldsymbol{z} \mid \mathcal{S}) \cdot p(\mathcal{S}) \\
& =k \cdot p\left(\boldsymbol{z} \mid \mathcal{Q},\left\{\hat{\boldsymbol{\theta}}^{M L}(\mathcal{Q})\right\}\right) \cdot p(\mathcal{Q}) .
\end{aligned}
$$

We assume the texture processes of the individual regions to be 'pairwise statistically independent'; this denotes a situation where the knowledge of the complete texture signal inside region $R_{i}$ does not provide any information on the texture signal inside region $R_{j}, j \neq i$. Under this assumption, the joint probability density of observing all the texture signals - in other words: the complete vector-valued image $\boldsymbol{z}$ - can be factorized in

$$
p(\boldsymbol{z} \mid \mathcal{Q})=\prod_{R_{i}} \prod_{k} p\left(\boldsymbol{z}_{i k} \mid \hat{\boldsymbol{\theta}}_{i}^{M L}\right),
$$

where index $i$ enumerates the regions, and index $k$ runs over the feature channels. The simple structure of eq. 6] does of course result from the assumed region-toregion and inter-feature channel independences stated in assumptions A1 and A2. 


\section{The Contour Relaxation}

With the target function to be maximized being defined by the preceding section, we proceed now to present the mere optimization method. We begin with an initial partition $Q_{0}$ and improve it by a constrained variation of pixel labels. We regard only those grid points $x_{0}$ that are located on the contour of a region thus the notion 'contour relaxation' - , and check whether certain changes of the point's region label yields an increase of criterion (3). If so, that particular label change is executed. However, only the current label $q\left(x_{0}\right)$, and the labels of the eight nearest neighbours of $x_{0}$ are allowed for $x_{0}$. Since the labels of all other pixels are kept fixed in each step, only those 8 cliques that include $x_{0}$ have to be taken into account, as only their potentials depend on the label of $x_{0}$. Thus, the 'label image term' $p(\mathcal{Q})($ Eq. 4) can be written as

$$
p(\mathcal{Q})=k_{1} \cdot \exp \left(-n_{B}^{\prime} B-n_{C}^{\prime} C\right) .
$$

Here, $k_{1}$ is a constant, and $B$ and $C$ are the costs for inhomogeneous horizontal/vertical, and diagonal cliques, respectively. The exponential term implicitly varies with the choice of the label for $x_{0}$ : the numbers of inhomogeneous cliques in the regarded clique subset for different choices of $q\left(x_{0}\right)$ are expressed by the values $n_{B}^{\prime}$ and $n_{C}^{\prime}$.

Correspondingly, the conditional likelihood of the image data $\boldsymbol{z}$ given the partition $\mathcal{Q}$ can be expressed as the product of a constant term and a variable term that depends on $q\left(x_{0}\right)$ :

$$
p(\boldsymbol{z} \mid \mathcal{Q})=k_{2} \cdot \prod_{\left\{\mathcal{R}_{j}\right\}} p\left(\boldsymbol{z}\left(\mathcal{R}_{j}\right) \mid \boldsymbol{\theta}\left(\mathcal{R}_{j}\right)\right) .
$$

Note that the product on the rhs comprises only those regions $R_{j}$ that are neighbors to site $x_{0}$, or directly include contour pixel $x_{0}$. It is important that the ML estimates of the region parameters change depending on the choice of the label chosen for $x_{0}$. This all boils down to evaluating the expression

$$
p(\boldsymbol{z}, \mathcal{Q})=k_{1} \cdot k_{2} \cdot \exp \left(-n_{B}^{\prime} B-n_{C}^{\prime} C\right) \cdot \prod_{\left\{\mathcal{R}_{j}\right\}} p\left(\boldsymbol{z}\left(\mathcal{R}_{j}\right) \mid \boldsymbol{\theta}\left(\mathcal{R}_{j}\right)\right),
$$

for all the (few) legal choices of $q\left(x_{0}\right)$. Grid point $x_{0}$ obtains the specific label that maximizes expression (9). In a practical implementation, the negative logarithm of equation (9) is minimized, which directly yields an energy function that has been constructed from 'first principles'.

A simple, though efficient optimization scheme is obtained by scanning the image several times, with systematically varying the scan direction each time. Since the presented optimization operation needs only to be executed for those grid points that are located on the boundary of a region, computational costs are low. The values of the parameters $B$ and $C$ of the Gibbs model are far from being critical; in our experiments, $B$ had values between 0.3 and 1.5 (typically 0.7 ) and $C=B / \sqrt{2}$. The relaxation may be stopped as soon as a scan over the whole image yields only few label changes. In general, about 2-4 iterations are required. 


\section{$5 \quad$ Likelihood Expressions for Region-Oriented Segmentation Approaches}

Under different pdf models (Gaussian, Laplacian, unilateral exponential...), the maximum likelihood estimates of the pdf parameters only depend on some few simple functions of the observables $z_{j}$ that need to be updated continuously:

$-N \stackrel{\text { def }}{=} \sum_{j} 1, \quad$ the number of measurements in the cell.

$-S \stackrel{\text { def }}{=} \sum_{j} z_{j}$, the sum of all measurement values.

$-Q \stackrel{\text { def }}{=} \sum_{j} z_{j}^{2}$, the sum of the squares of all measurement values.

$-A \stackrel{\text { def }}{=} \sum_{j}\left|z_{j}\right|, \quad$ the sum of the absolute values of all measurement values.

For a given region, and assuming that the measurement values are uncorrelated between channels, only these numbers have to be computed (per feature channel), and only these numbers are needed for the computation of the likelihood terms appearing in eq. (8) and (9). Since, under a given pdf assumption, these numbers fully represent all the necessary information contained in a set of measurement values, these numbers are denoted as 'sufficient statistics'.

\subsection{The Data Likelihood Term for a Set of Measurements under the Gaussian Assumption}

The value of the pdf for $N$ statistically independent scalar measurements $z_{j}$ from the same Gaussian distribution is

$$
p_{z}(\boldsymbol{z})=\left(\frac{1}{\sqrt{2 \pi}}\right)^{N} \cdot\left(\frac{1}{\sigma}\right)^{N} \cdot \exp \left(-\frac{1}{2} \sum_{j} \frac{\left(z_{j}-m_{i}\right)^{2}}{\sigma^{2}}\right) .
$$

Estimating the parameters and finding the likelihood value. For the parameters $m$ and $\sigma^{2}$, we assume their maximum likelihood estimates are determined from the given data set. We obtain

$$
\hat{m}=S / N ; \quad \hat{\sigma}^{2}=\frac{Q}{N}-\left(\frac{S}{N}\right)^{2} .
$$

With these estimates, the exponent in eq. 10 is

$$
\frac{1}{2 \sigma^{2}} \sum_{j}\left(z_{j}-\hat{m}\right)^{2}=\frac{N}{2} .
$$

Inserting this into eq. 10, we obtain

$$
p_{z}(\boldsymbol{z})=\left(\frac{1}{\sqrt{2 \pi}}\right)^{N} \cdot\left(\frac{N^{2}}{N Q-S^{2}}\right)^{N / 2} \cdot \exp \left(-\frac{N}{2}\right)
$$


Taking the logarithm of this expression, we obtain

$$
\ln p_{z}(\boldsymbol{z})=\frac{N}{2} \cdot\left(-\ln (2 \pi)+\ln \left(\frac{N^{2}}{N Q-S^{2}}\right)-1\right) .
$$

Data likelihood terms for other pdf models, e.g. Laplacian can be determined easily and used in exactly the same way.
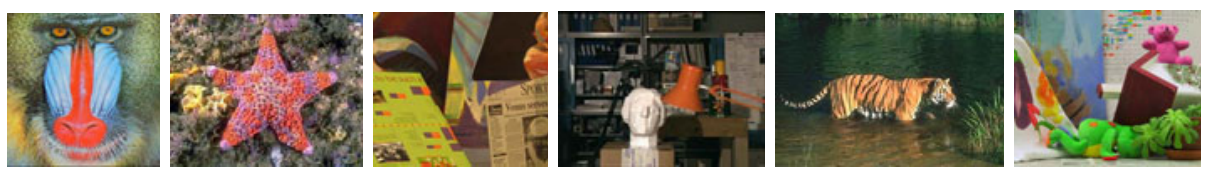

Fig. 3. Original images used within the experiments: (left to right) Baboon, Starfish, Venus, Tsukuba, Tiger and Teddy

\section{Experimental Results}

In the following we present experimental results obtained for multi-channel contour relaxation applied to single images and to a stream of images. We show results obtained when the initial segmentation is given by (i) a blind segmentation or (ii) a sparse segmentation, i.e., in the simplest case provided as several strokes in the image marking the desired foreground and background. Figure 4 (top left) and Fig.7 (bottom left) visualize exemplary initial segmentations for both cases.

For images with a resolution of $640 \times 480$ pixels, 4 passes of a $\mathrm{C} / \mathrm{C}++$ version of the contour relaxation on the three color channels takes about 150 milliseconds on an Intel Xenon $2.8 \mathrm{GHz}$ processor. Note that this implementation is only single threaded and unoptimized.

\subsection{Contour Relaxation Applied to Single Images}

In this section, we show segmentation results obtained when multi channel contour relaxation is applied to single images. We use several well known images from the Middlebury web site [8] and the Berkeley segmentation database [10] (cf. Fig.3).

Automatic Initialization by a Blind Segmentation. A blind segmentation is generated by subdividing the input image into non-overlapping regions of $n \times n$ pixels, where $n=32$ throughout the experiments. The clique costs are set to 0.3 and $\frac{0.3}{\sqrt{2}}$ for inhomogenous horizontal or vertical and diagonal cliques, respectively. Then, contour relaxation is applied to the initial segmentation where first the sufficient statistics of each region on each of the three image channels 

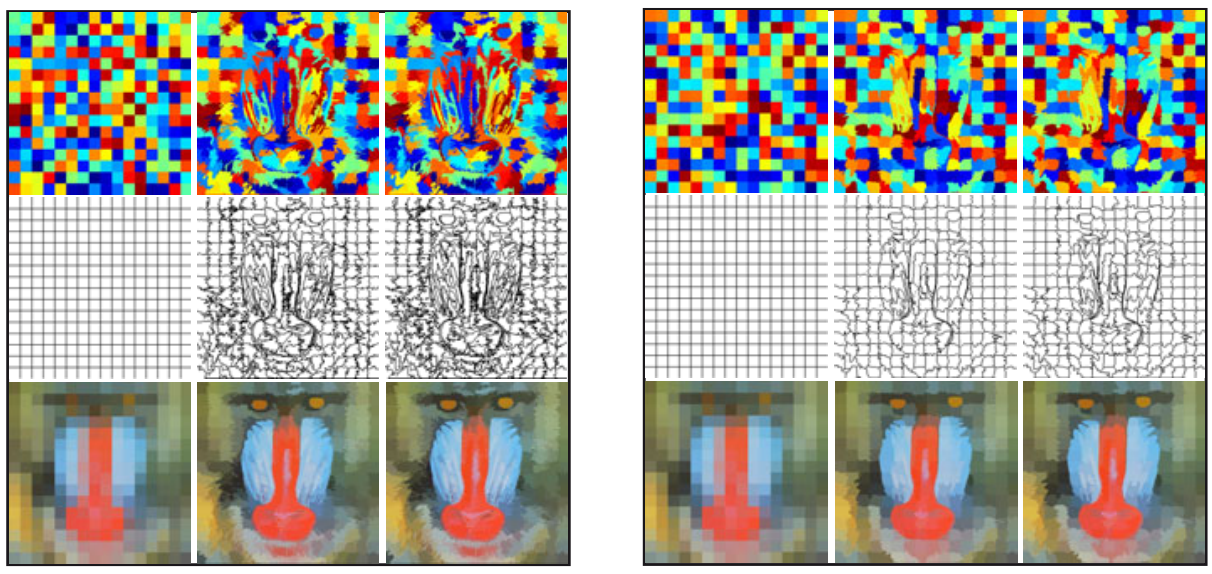

Fig. 4. Segmentation results for image 'Baboon': (left) low clique costs, (right) high clique costs. Upper row: label images after 0,4 and 8 runs of contour relaxation. Middle row: contour images; lower row: region mean images. Best viewed in color.

$(\mathrm{Y}, \mathrm{U}, \mathrm{V}) 2$ are computed. Then the relabeling of pixels along the region contours is performed as described in Sec.4. We perform 4 passes of contour relaxation, where each pass is subdivided into 4 sub-passes, that is, within the relabeling algorithm, pixels are visited from (i) left-right, up-down, (ii) left-right, down-up, (iii) right-left, up-down and (iiii) right-left, down-up. The purpose of this scheme, also known as using coding sets, is to bias the results as little as possible with respect to the used pixel visiting scheme. For all presented results, at latest after 4 passes of contour relaxation have been performed, the segmentation remained stable, e.g., no further significant change of the labeling could be observed.

Figures 4 to 6 show the results for the data sets Baboon, Venus, Tsukuba, Cones and Teddy. Here, we display the label arrays, the contour images and the region mean images after 0,4 and 8 sub-passes of contour relaxation have been performed. The region mean images are generated by filling a region of the label array with the color mean value of all pixels assigned to that specific label (cf. Sec.55).

The result figures show that our approach typically produces an over-segmentation in the sense of a super-pixel representation of the input image. That is, the image is subdivided into many regions (super-pixels), where all pixels in one region are similar with respect to their color value. Here, it is of utmost importance that a single super-pixel does not contain different objects within the image, such as foreground and background, and that the super-pixels are aligned with the object boundaries. Depending on the number of labels (i.e., the number of regions $R_{n}$ ) within the initial blind segmentation, the amount of over-

${ }^{2}$ The Y channel encodes the luminance, $\mathrm{U}$ and $\mathrm{V}$ the chrominance of each pixel. This color space is in reasonable accordance to the assumption of statistically independent feature channels, since it decorrelates the (strongly correlated) RGB color values. 

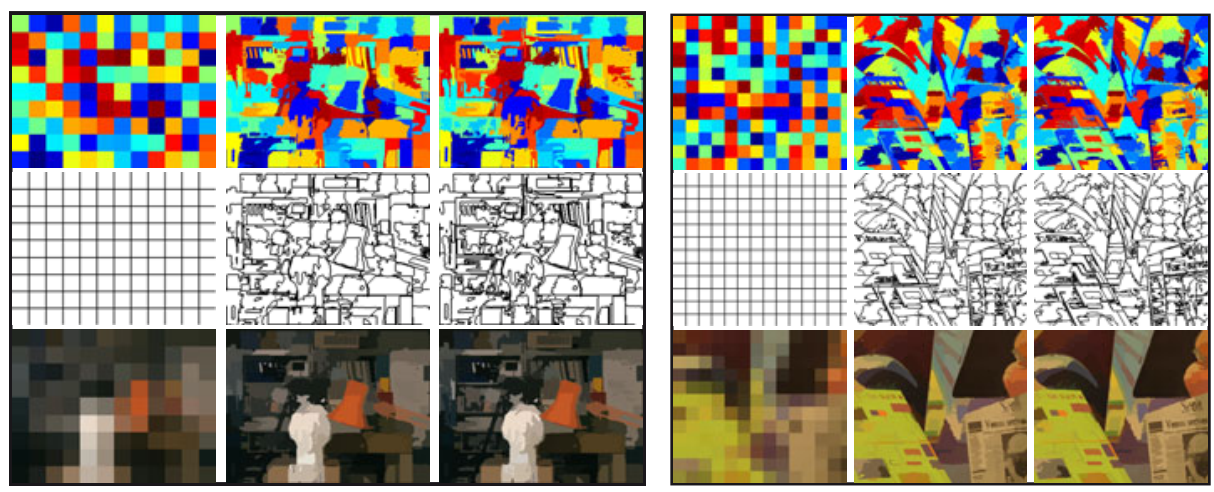

Fig. 5. Segmentation results for image 'Tsukuba' (left) and image 'Venus' (right): Upper row: label images after 0,4 and 8 runs of contour relaxation. Middle row: contour images; lower row: region mean images. Best viewed in color.

segmentation can be controlled. However, as long as only low-level information, such as the pixel color value, is available, in general no segmentation on an object level can be computed, as typically mid-level information, such as motion or depth, or high-level object knowledge are decisive.

Throughout Fig. 4 to Fig.6 it can be seen that the super-pixels are aligned with the object boundaries, such as the head in the Tsukuba image, the posters and newspaper in the Venus image, the tail of the tiger within the Tiger image (cf. with the results obtained in [3]) and the cuddly toys within the Teddy image. Note that also fine-grained details, such as the characters on the newspaper within Venus, or the Baboons whiskery, are captured as well. For the Baboon image we also show the influence of different clique costs on the resulting segmentation. In Fig.4 (right) the costs for inhomogeneous cliques are doubled. Therefore the segmentation appears to be more block-like, whereas in the case for lower clique costs (Fig.4 (left)), the segmentation adapts more to the fine-grained details. This can also be seen within the contour images for both cases, where the contour image in the case of higher clique costs has a much less 'ragged' appearance.

Manual Initialization by a Given Sparse Segmentation. The results presented so far have been obtained in a completely unsupervised manner, where the initial segmentation was generated 'automatically'. For a typical task such as foreground/background segmentation, often some kind of user input is available, where parts of the foreground and parts of the background are explicitly labeled. Such user input can be given by several line strokes within the input image, marking the different textures (or objects) to be segmented out. Figure 7 shows an example for the Starfish image, where the background is marked by a blue stroke and the foreground object by a red stroke. All other pixels remain initially unlabeled. We use the same initial segmentation as in [13]. 

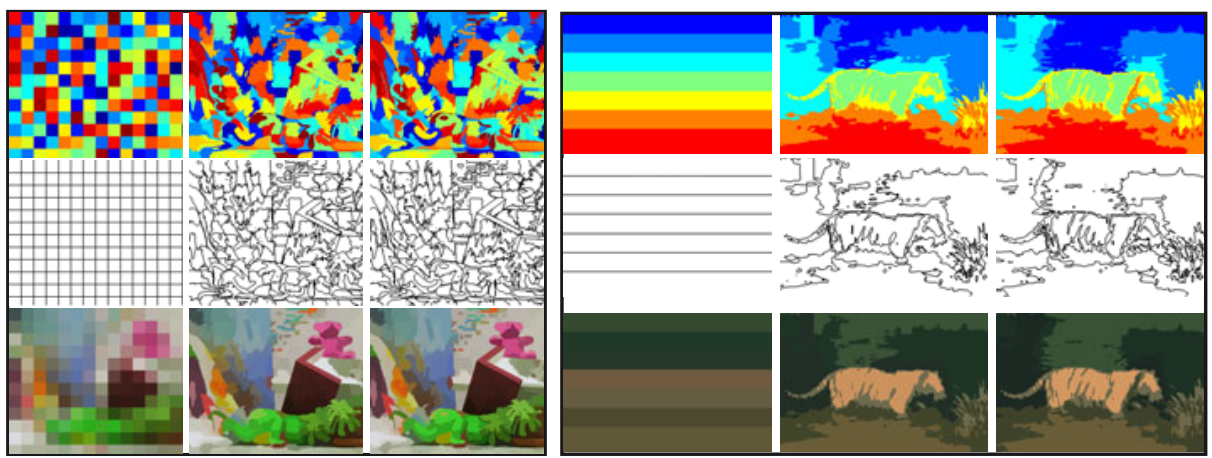

Fig. 6. Segmentation results for image 'Teddy' (left) and 'Tiger' (right): Upper row: label images after 0,4 and 8 runs of contour relaxation. Middle row: contour images; lower row: region mean images. Best viewed in color.
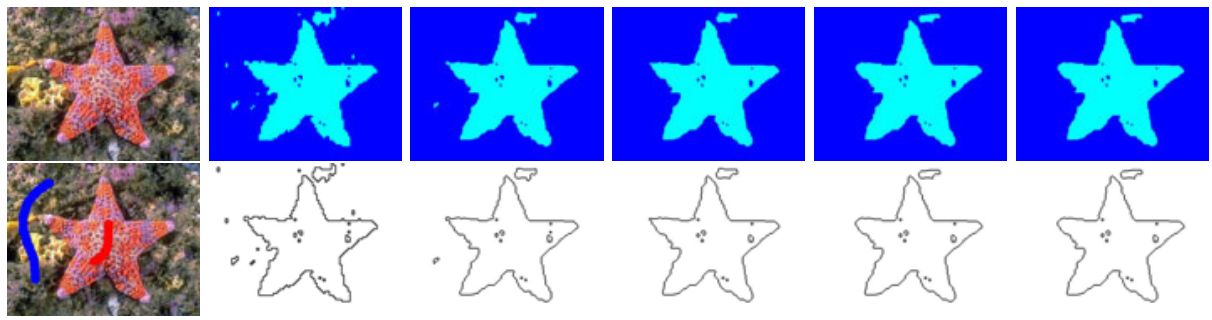

Fig. 7. Segmentation of the 'Starfish'. First column shows the original image and the user strokes. Second column shows the result of a maximum likelihood classification, columns 3 to 6 show results after 2, 4, 8 and 12 passes of contour relaxation, where row 1 is the label image and row 2 the contour image. Best viewed in color.

We obtain a full segmentation as described in Sec.4 by a maximum likelihood classification of all unlabeled pixels to either foreground or background. Such maximum likelihood labeling is then subject to several passes of contour relaxation, to remove false classifications and to smooth the label image. The second column of Fig.7 7 shows the maximum likelihood classification result for the Starfish image and the corresponding contour image. This yields a label array where the contours of the foreground object are already well captured. However, several pixels which lie in the background are classified as foreground and vice versa. Columns 3 to 6 in Fig. 7 visualize how the segmentation is adapted by applying several passes of multi channel contour relaxation. We are aware of the fact that optimum solutions to such two-class labeling problems are available 132 . However, we would like to stress that the contour relaxation gives results which are virtually indistinguishable from these optimum ones, even for multi-class problems, at much lower complexity of the procedure and lower computational effort. It can be seen that a small amount of pixels in the upper right 
are still marked as foreground. This is caused by the fact that the statistics of the three color channels for this specific region and the foreground object are nearly identical. Resolving this issue requires higher level information.

\subsection{Temporal Propagation of the Segmentation}

The experiments described so far were applied to single images only. In the following, we show briefly that our approach can be applied to image sequence segmentation as well.

In order to let image sequence segmentation be efficient it is crucial to exploit prior knowledge obtained during the processing of the previous images. At time $t$, such prior knowledge is, e.g., the segmentation result obtained at time $t-1$. In our framework, the segmentation result from time $t-1$ will therefore be the initialization of the segmentation to be computed for the image at time $t$. This significantly decreases the computational effort. Furthermore the labelings of the images are to a very large extent coherent over time, i.e., a single object will almost always maintain its label from image to image. Figure 8 shows the segmentation results obtained for a cluttered office scene, where a person is moving in front of the camera. The segmentation was automatically initialized with a blind segmentation as described earlier. Then, 4 passes of contour relaxation have been applied and the resulting segmentation was used as the initialization for the next frame to be processed. Again, the label and the contour images show the desired feature of super-pixels being well aligned with object boundaries. Fine grained object structures such as the table legs, or the lamp are well preserved. Furthermore, the labeling remains largely consistent over time.
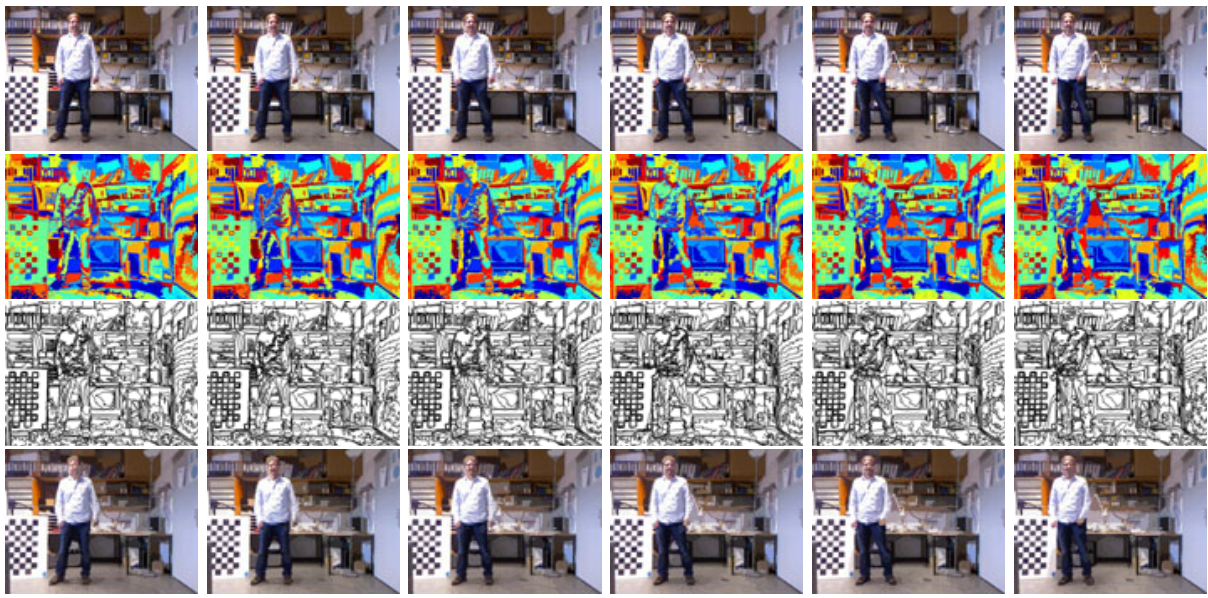

Fig. 8. Segmentation results for cluttered office scene: (1. row) label images, (2. row) contour images, (3. row) region mean images for frames 185, 188, 191, 194, 197 and 200 where the label images are propagated. Best viewed in color and upscaled. 


\section{Conclusions}

We have demonstrated the performance of the combination of a region-based stochastic model with the 'contour relaxation' optimization which very efficiently yields a 'superpixel' segmentation. Even though we are fully aware that no guarantees concerning achieving a globally optimum solution can be given, the various application examples hopefully conveyed the very 'benign' performance and the versatile applicability of the scheme. The generalization of the approach to texture, or using depth and/or motion is obviously a promising next goal.

\section{References}

1. Achanta, R., Shaji, A., Smith, K., Lucchi, A., Fua, P., Suesstrunk, S.: SLIC Superpixels. Tech. Rep. Nr. 149300, EPFL, Lausanne (CH) (June 2010)

2. Boykov, Y., Veksler, O., Zabih, R.: Fast approximate energy minimization via graph cuts. IEEE Trans. PAMI 23(11), 1222-1239 (2002)

3. Brox, T., Cremers, D.: On local region models and a statistical interpretation of the piecewise smooth Mumford-Shah functional. IJCV 84(2), 184-193 (2009)

4. Chellappa, R., Chatterjee, S.: Classification of textures using Gaussian Markov random fields. IEEE Trans. ASSP 33(4), 959-963 (1985)

5. Cremers, D., Rousson, M., Deriche, R.: A review of statistical approaches to level set segmentation: integrating color, texture, motion and shape. International Journal of Computer Vision 72(2), 195-215 (2007)

6. Derin, H., Cole, W.: Segmentation of textured images using Gibbs random fields. Computer Vision, Graphics, and Image Processing, pp. 72-98 (1986)

7. Felzenszwalb, P., Huttenlocher, D.: Efficient graph-based image segmentation. IJCV 59(2), 167-181 (2004)

8. http://vision.middlebury.edu

9. Levinshtein, A., Stere, A., Kutulakos, K., Fleet, D., Dickinson, S., Siddiqi, K.: Turbopixels: Fast superpixels using geometric flows. PAMI 31(12), 2290-2297 (2009)

10. Martin, D., Fowlkes, C., Tal, D., Malik, J.: A database of human segmented natural images and its application to evaluating segmentation algorithms and measuring ecological statistics. In: Proc. ICCV 2001, vol. 2, pp. 416-423 (2001)

11. Mester, R., Franke, U.: Statistical model based image segmentation using region growing, contour relaxation and classification. In: Proc. SPIE Visual Communications and Image Processing, Cambridge, MA, pp. 616-624 (1988)

12. Ren, X.R., Malik, J.: Learning a classification model for segmentation. In: Proc. ICCV, vol. 1, pp. 10-17. IEEE Computer Society Press, Los Alamitos (2003)

13. Rother, C.: Tutorial on map inference in discrete models. In: ICCV (2009)

14. Sclove, S.L.: Application of the conditional population-mixture model to image segmentation. IEEE Trans. PAMI 5, 428-433 (1983)

15. Therrien, C.W.: An estimation-theoretic approach to terrain image segmentation. Computer Vision, Graphics, and Image Processing 22(3), 313-326 (1983)

16. Unnikrishnan, R., Pantofaru, C., Hebert, M.: Toward objective evaluation of image segmentation algorithms. IEEE Trans. PAMI, 929-944 (2007)

17. Veksler, O., Boykov, Y., Mehrani, P.: Superpixels and supervoxels in an energy optimization framework. In: Daniilidis, K., Maragos, P., Paragios, N. (eds.) ECCV 2010. LNCS, vol. 6315, pp. 211-224. Springer, Heidelberg (2010) 